\title{
Current Problems and Improvements in Anatomy Teaching
}

\author{
Li MA \\ Shaanxi University of Traditional Chinese Medicine, Xianyang, Shaanxi, China
}

\begin{abstract}
Keywords: Anatomy Teaching; Clinical Thinking; Current Problems; Teaching Improvements.
\end{abstract}
\begin{abstract}
The purpose of education is not only to teach students how to master basic medical knowledge, but also to help them develop scientific thinking methods, and master comprehensive clinical skills and practical ability. This article explores the current problems in anatomy teaching, and then probes the improvements to strengthen operation ability of medical students, raises the teaching quality of anatomy, and lays a solid foundation for clinical course learning.
\end{abstract}

\section{Introduction}

As medical backbone courses of human anatomy, the teaching mode has experienced a long and arduous road of the reform. However, as the scale of higher education in our school, with school level running and education tasks rising gradually, the existing problems in anatomy teaching are found.

\section{Ignoring the Importance of Clinical Practice in the Teaching Content}

Anatomy "at present, the system of teaching material version although many, but most of the textbooks in the aspect of content arrangement basic same, theoretical interpretation is heavy, light the phenomenon of clinical application, a lack of theory with practice of clinical cases, single teaching contents. In addition, due to no longer contact clinical full-time teachers, of clinical knowledge more and more unfamiliar, inevitably leads to most of the teachers in the teaching process focus on "system's anatomy" the interpretation of the basic theoretical knowledge and teaching, and neglected the theory knowledge and the relationship between clinical, makes the students cannot be learned theoretical knowledge and clinical practice, cannot form the clinical thinking, there is a big passive learning, teaching effect is not ideal. Our teaching and research section in part and clinical professional class discussion, most of the students to realize the importance of systematic anatomy study, think that as long as remember the basic morphological structure, can pass the final exam, lead to the implicit learning and passivity, eventually cannot be learned knowledge to achieve mastery through a comprehensive study, this will follow-up clinical professional course for students learning and practice."System's anatomy" and how to break through the traditional teaching mode in the teaching in the teaching process can not only strengthen the basic theory of systematic anatomy knowledge, train the students' active and combining the basic theory and clinical cases, these have become the "system's anatomy" need thinking to solve the problems in the process of teaching.

\section{Abstraction and Rigidity in Class}

"Systematic anatomy" belongs to the morphological discipline. Each chapter focuses on the description of the morphology and location of different systems. From each system, each organ to each morphological structure and location of the detailed description, to achieve a comprehensive understanding of the content of each chapter, the difficulty is conceivable. There are many contents and heavy tasks. If the content of teaching materials is taught comprehensively, the time of study will not be enough. If teachers in the teaching practice, the diversity and does not pay attention to the flexible use of teaching method, can make the students feel boring, rigid formality, drowsy, unable to understand the implied deep mechanism, easy to produce the fear, study interest is not high, the gruff recite to cope with the tests, which affect the teaching quality, causes the teaching effect is not ideal. 


\section{Still Corresponding Problems in Teaching Model}

In the teachers' teaching as the main system anatomy "on the traditional teaching mode, teaching team we tried" based on the teaching of learning "as the foundation, joint" problem based learning "teaching and the comprehensive teaching mode of" case teaching ", supplemented by interactive teaching, multimedia teaching and bilingual teaching of diversified teaching mode reform, has produced results, but there are still problems accordingly.

1) The PBL teaching PBL teaching in our university of traditional Chinese medicine (TCM) clinical, and other professional has been used in the systematic anatomy teaching, and in the teaching, we found that the simple PBL teaching, there is still evident: 1. the student aspect of knowledge narrow, especially early classes, students new to medicine, they know very little about anatomy, more do not understand the related medical knowledge, the anatomy knowledge can only be accumulation increased progressively with courses, students knowledge has apparent simplicity, if written discussion topic is more complex, involving the content of the other basic medicine and clinical disciplines, students are hard to form their own thinking, not highlight learner centered learning situation and its effect, the effect of the discussion is often poor. Even if a student finds an answer by looking up information, he or she knows nothing about it. 2 . There is a serious shortage of teaching resources.PBL is a kind of higher requirements for teachers' quality of teaching methods, teachers need to not only familiar with the contents of this discipline, and other related disciplines of knowledge, it will take more time and energy, teachers, and most of the teachers' workload of this discipline is larger, there is no time to research and discussion of PBL teaching, further improve the quality of teachers. 3. There are insufficient teaching conditions. PBL teaching process requires a lot of resources, such as library collection, a large number of literature database, the proper teaching materials and special classrooms and teaching AIDS and other hardware facilities, the condition is the guarantee of students' learning attitude and learning effect, this requires schools to support and investment. 4. As an important basic course for medical students, teaching quality directly affects the training quality of students. Anatomical terminology, medical terminology, hard to remember difficult hard to understand, if the extensive use of PBL teaching in teaching, students are likely to focus on problem solving and lose the opportunity to learn basic knowledge of anatomy, will cause the anatomy knowledge is not enough, it is unfavorable to the development of medical students.

2) Case teaching method in the teaching of anatomy, use case analysis to clinical knowledge and the basic knowledge of closely linked, so that the students fully realize the importance of learning basic knowledge of anatomy. This teaching method greatly improves students' initiative in learning and improves the teaching effect. Case teaching is the premise of successful implementation requires students to have certain medical knowledge, the basis of the teaching object is just contact us two systematic anatomy and biology medical foundation course of freshman students, how to successfully carry out case teaching is an important challenge for our teachers, so the choice of cases and design is the key to success: case should have distinct pertinence, the case should be closely linked with the content of courses, students can use the have learned the knowledge analysis case, establish the clinical thinking ability of students; We should select cases of common diseases with typical symptoms, which can introduce basic knowledge more easily and clearly without excessive expansion of basic knowledge. Attach importance to scientific design of problems. Teachers refer to a large number of relevant knowledge of problems and combine basic knowledge and clinical knowledge before teaching. In a word, the selection of successful cases not only avoids the negative influence of rote memorization in traditional anatomy teaching, but also facilitates students to clearly understand and master the key and difficult knowledge of basic knowledge. Similarly, this kind of teaching method also faces the same series of problems of PBL teaching.

3) Multimedia teaching: since school multimedia classroom use of multimedia teaching mode into the system of anatomy teaching, under the joint efforts of our teaching team, and have a lot of animation produced excellent pictures and texts PPT courseware. Abundant pictures and intuitive animations have solved some difficult problems that are difficult to understand and won unanimous praise from students. However, there is a conflict between understanding the classroom content and 
taking good class notes. When students are busy taking notes, they ignore the teacher's analysis of key problems, so that they do not fully understand the classroom content. Students who listened carefully did not have time to take good notes and could not guide their future review. This is a common problem in multimedia teaching and it must be solved.

\section{Simplicity of the Assessment and Evaluation Method}

Anatomy of the current system evaluation method mainly adopting the combination of test scores and normal operation, the overall $=$ test scores by $70 \%+30 \% \mathrm{x}$ grade at ordinary times, pay attention to the students understand the degree of memory testing of outline content, lack of practical application, the basic experiment skills of operation, so when students after learning this course though obtained higher grades through mechanical memory, but the actual master did not meet the expected requirements of knowledge and skills, foundation weak, the resistance is larger in the subsequent course of study. On the basis of theory and experiment teaching content reform to change old exam evaluation system, and truly improve the students' scientific research ability, as soon as possible into the role of medical students, cultivate medical thinking ability.

To avoid flat narration, abstraction and rigidity, improve students' learning initiative and enthusiasm, and cultivate the clinical thinking ability, the following measures are carried out.

\subsection{In anatomical teaching content}

We use the syllabus and compile for universal use materials as the basic teaching content, delete the old and backward content, adding new content, with close links to the clinical increased dynamic and development trend of this new research expanded student aspect of knowledge, preliminary training of their clinical thinking ability, laid a more solid foundation for their future clinical study.

\subsection{In teaching resources of forensic anatomy}

Anatomic teaching resources include clinical cases, computer anatomy questions, anatomic picture video library, anatomic specimens and models. Continuous screening in recent years, we tried to use many ways to collect all kinds of good cases, constantly enrich and optimize the cases, gradually set up its own clinical cases, to anatomy teaching for middle school students clinical thinking ability. We will organize the teaching experienced teachers proposition, increase comprehensive analysis topic quantity, less memory topic quantity, speed up the construction and optimization of computer exam, pay attention to the assessment of students' clinical thinking ability, improve the quality of teaching evaluation. At the same time, we strive to improve the video library of anatomical images for theoretical and experimental teaching and assessment. In addition, new cadaver specimens are made and purchased continuously, and the specimens are classified and preserved for experimental teaching.

\subsection{In the teaching methods of anatomy}

We will take the teacher as the leadership, take the student as the main body, to clinical problems as the center, the use of various teaching methods, including heuristic, discussion-based to replace the traditional "force-feeding" teaching methods, inspire the student to the basic theory with clinical practice, stimulate students' active thinking, gradually cultivate the students' clinical thinking ability, lay the foundation for subsequent courses. In the anatomy experiment lesson, we will pick up the corresponding bodies from the pictures and videos library operation video or video playback of clinical surgery, let the students learn the structure of fresh specimens, such as observing abdominal surgery, the ministries of the layers of skin incision structure is different, more deeply know and understand the distinguished structure, master the basic theory. In addition, in the anatomy experiment class, we will boldly reduce the verification experiment and carry out the open experiment. By teachers lead students to do research, to observe and measure the anatomical structure of clinically relevant, provide important anatomic data for clinical, at the same time the organic combination of anatomy and clinical knowledge, deepened the understanding of classroom 
knowledge, enhance the enthusiasm of the students' interest in learning and exploring the unknown, training the students' clinical thinking ability.

\subsection{In comprehensive quality of teachers}

As the saying goes, "a good teacher makes a good teacher.'The breadth and narrow of teachers' knowledge structure and the level of intelligence are directly related to the improvement of teaching quality and the cultivation of talents. Cultivate the students' clinical thinking ability, improve medical students' comprehensive quality, must want to have a strong sense of teaching, high business level, strong innovation consciousness, enterprising, dedication of teachers. For this, for many years teaching and research section I advocate and encourage the teachers to strengthen continue to education itself, in the next urgent hope to be able to regularly participate in new technology, new knowledge, such as continuing medical education training, in order to broaden the knowledge; Strive for all teachers to participate in various academic activities, be able to actively carry out scientific research activities and timely track the development of the discipline at home and abroad; Regular reading report activities will be held, class preparation, teaching plan discussion and discussion on the reform of teaching content, methods and means will be carried out, and the teaching level will be continuously improved. Through various aspects of training and learning, improve the comprehensive quality of teachers. At the same time, teachers keep learning various clinical cases, and increase the clinical knowledge related to anatomy. Consult with the clinician at any time and strive to deepen the understanding and mastery of relevant clinical knowledge. At any time, typical cases reported in the press and daily life will be added to the case textbook library; When preparing the clever and the clinical knowledge properly into the corresponding teaching content, make the anatomy knowledge more interesting, informative, and applicability, for future basic and clinical knowledge learning and clinical work to lay a solid foundation.

\subsection{In the clinical practical skills}

In medical education, teaching practical skills is, accurate, comprehensive and mastering clinical examination the best way to diagnosis and operation skills, and anatomy is a practical very strong cohesion and clinical medicine of "bridge". With the deepening of teaching reform, anatomy has been gradually transformed from a basic medical course to a basic clinical course. Based on this, my teaching and research section in the system based on the theory of anatomical knowledge, increase the related clinical skills operation knowledge content, such as punctures, urethral catheterization, and detailed about the related skills to the local anatomic structure characteristics, operation main point and the matters needing attention. Extension to expand traditional theory teaching clinical skills, stimulate students interest in learning, the close ties between the clinical practice, guide the students into the process of active learning, promote students' skills of operating points of understanding and mastering, improve the ability of clinical practice. Lumbar puncture demo specimens, for example, reflect puncture and each hierarchy between anterior cruciate ligament (skin - spines on the spine ligament previously - the ligament flavum - periosteum arachnoid - subarachnoid), causes the student to intuitive understanding of related anatomical basis, clinical practice and contact operation main point and the matters needing attention. At the same time of correctly expressing the anatomical structure, appropriate extension is made to the clinical application, so as to help students acquire relevant knowledge of clinical application from the specimen, and give clues and references for thinking problems.

\subsection{In the examination and assessment system}

A multi - form course evaluation system of system anatomy was established. In order to avoid the traditional single final exams to evaluate teacher's teaching effect and the disadvantages of student learning, new way of appraisal, pay attention to students in theory, experiment and comprehensive abilities of medical record assignments, group discussions, improve the examination score, make its accounted for up to $50-60 \%$, decrease the knowledge score to $40-50 \%$, and is objective and fair evaluation of students to master the ability of knowledge and to analyze and solve the problem of 
the comprehensive quality.

\section{Summary}

Through optimizing the system anatomy "anatomy teaching contents, reform teaching methods, improve the anatomy teaching method, pay attention to the clinical practical skills and the combination of anatomy teaching, cultivate students' operation ability, and build more forms of the curriculum evaluation system anatomy method, from the establishment of a more forms, multi-channel, omni-directional mode of diversified teaching, dominated by the teacher, the students as the main body of study way, fully mobilize the enthusiasm of students, causes the student to make a comprehensive analysis of the specific clinical phenomenon, logic reasoning, conducive to the combination of knowledge, ability, training students clinical thinking ability, Lay the foundation for subsequent courses. Systematic anatomy of the reform of experiment content and evaluation methods, breaking the traditional simple combination of theory and experiment, to make the students actively participate in the learning process, emphasizes the solution of the problem, rather than to acquire knowledge, students in solving problems in the process of learning the necessary knowledge, learn to correct clinical thinking, improve the students' practical skills, stimulate students' operation ability, and truly objective and fair evaluation of the ability of students to master knowledge and analyze and solve problems of the comprehensive quality.

\section{References}

[1] Yingfeng Wu. A Comparison Between Problem-based Learning and Traditional Teaching Model [J]. Foreign Medicine, 1995 (03): 1-4.

[2] Baichun Yao, etc. The Practice and Thinking of PBL Teaching Model in Regional Anatomy Teaching [J]. Northwestern Medicine Education, 2008 (05): 926-927.

[3] Shangqing Liu, etc. The Exploration of Cultivating Clinical Thinking Ability of Medical Students in Human Anatomy Teaching [J]. Journal of North Sichuan Medical College, 2007 (04): 400-402.

[4] Xinmei Hu. On The Cultivation of College Students' Independent Learning Ability [J]. Education and Occupation, 2015 (27): 110-112. 